\title{
Deep optical observations of the supernova remnants G 126.2+1.6, G 59.8+1.2 and G 54.4-0.3
}

\author{
P. Boumis ${ }^{1}$, F. Mavromatakis ${ }^{2,3}$, E. M. Xilouris ${ }^{1}$, J. Alikakos ${ }^{1,4}$, M. P. Redman ${ }^{5}$, and C. D. Goudis ${ }^{1,4}$ \\ ${ }^{1}$ Institute of Astronomy \& Astrophysics, National Observatory of Athens, I. Metaxa \& V. Paulou, P. Penteli, 15236 Athens, Greece \\ 2 University of Crete, Physics Department, PO Box 2208, 71003 Heraklion, Crete, Greece \\ 3 Technological Education Institute of Crete, General Department of Applied Science, PO Box 1939, 71004 Heraklion, Crete, Greece \\ 4 Astronomical Laboratory, Department of Physics, University of Patras, 26500 Rio-Patras, Greece \\ 5 Department of Physics, National University of Ireland Galway, Galway, Ireland
} e-mail: ptb@astro.noa.gr

Received 20 May 2005 / Accepted 21 July 2005

\begin{abstract}
Optical CCD imaging and spectroscopic observations of three supernova remnants are presented. Optical emission from G 54.4-0.3 and G 59.8+1.2 is detected for the first time, while the first flux calibrated CCD images of the supernova remnant G 126.2+1.6 were performed in the optical emission lines of $\mathrm{H} \alpha+[\mathrm{N} \mathrm{II}]$, [O III] and [S II]. A mixture of filamentary and diffuse structures is observed in $\mathrm{G} 54.4-0.3$ and G 59.8+1.2, mainly in $\mathrm{H} \alpha+[\mathrm{N}$ II], while the deep optical images of $\mathrm{G} 126.2+1.6$ reveal several new filamentary and diffuse structures inside the extent of the remnant as defined by its known radio emission. In all cases, the radio emission is found to be well correlated with the optical filaments. [O III] emission was not detected at G 54.4-0.3 and G 59.8+1.2 while in G 126.2+1.6, significant morphological differences between the low and medium ionization images are present suggesting incomplete shock structures. Deep long-slit spectra were taken at different positions of the remnants. Both the flux calibrated images and the long-slit spectra clearly show that the emission originates from shock-heated gas, while some spectra of G $126.2+1.6$ are characterized by large [O III]/H $\beta$ ratios. This remnant's [O III] flux suggests shock velocities into the interstellar "clouds" between 100 and $120 \mathrm{~km} \mathrm{~s}^{-1}$, while the [O III] absence in the other two remnants indicates slower shock velocities. For all remnants, the $[\mathrm{S}$ II $] \lambda \lambda 6716 / 6731$ ratio indicates electron densities below $600 \mathrm{~cm}^{-3}$ with particularly low densities for G 54.4-0.3 (below $50 \mathrm{~cm}^{-3}$ ). Finally, the $\mathrm{H} \alpha$ emission has been measured to be between 3.0 to $15.2 \times 10^{-17} \mathrm{erg} \mathrm{s}^{-1} \mathrm{~cm}^{-2} \operatorname{arcsec}^{-2}, 3.2 \times$ $10^{-17} \mathrm{erg} \mathrm{s}^{-1} \mathrm{~cm}^{-2} \operatorname{arcsec}^{-2}$ and between 6.5 to $16.8 \times 10^{-17} \mathrm{erg} \mathrm{s}^{-1} \mathrm{~cm}^{-2} \operatorname{arcsec}^{-2}$ for G 54.4-0.3, G 59.8+1.2 and G 126.2+1.6, respectively.
\end{abstract}

Key words. ISM: general - ISM: supernova remnants - ISM: individual objects: G 54.4-0.3, G 59.8+1.2, G 126.2+1.6

\section{Introduction}

The majority of the galactic supernova remnants (SNRs) have been identified by their synchrotron emission while some of the remnants have been discovered in the soft X-rays and in the optical band. Optical emission line observations of SNRs show that the $[\mathrm{S} \mathrm{II}] / \mathrm{H} \alpha$ ratio is typically higher than $\sim 0.4$, while in photoionized nebulae the ratio drops below 0.3-0.4 (Smith et al. 1993). Additional line ratios are used in doubtful cases.

The galactic supernova remnant G 126.2+1.6 was discovered in a $1420 \mathrm{MHz}$ radio continuum survey by Reich et al. (1979), where it appears as a large but not well defined shell ( $\sim 68^{\prime}$ in diameter) of low surface brightness. Radio observations were also performed at 408, 865, 1410, 2700 and $4850 \mathrm{MHz}$ by Reich et al. (2003), Joncas et al. (1989) and Fürst et al. (1984) and a spectral index of 0.5-0.6 was established. Optical filaments which coincide with the brightest radio emission on the west part of the remnant have been detected by Blair et al. (1980), Rosado (1982) and Fesen et al. (1983), while Xilouris et al. (1993) presented a wide field $\mathrm{H} \alpha+[\mathrm{N} \mathrm{II}]$ image $\left(26^{\prime} \times 40^{\prime}\right)$. In particular, imaging and spectroscopy of one of the filaments were performed by Blair et al. (1980) suggesting a large $[\mathrm{O} \mathrm{III}] / \mathrm{H} \beta$ ratio, while Rosado (1982) was found relatively bright filamentary emission in $\mathrm{H} \alpha$ and [S II], extending further to the north and south of the already known filaments. [O III] images of the west part of the remnant were also obtained by Fesen et al. (1983). These images show faint emission which appear correlated with the radio emission along the western rim. G 126.2+1.6 is classified as an old remnant which still shows strong [O III] emission (Raymond 1984).

The extended shell-type remnant G 54.4-0.3 was first identified by Holden \& Caswell (1969) during their $178 \mathrm{MHz}$ radio survey. Several radio observations have been performed (Junkes et al. 1992a and references therein) showning its nonthermal nature, a spectral index of $\sim 0.4$ and a nearly circular shell (with a gap near the north-east boundary). A distinct radio shell of $\sim 40^{\prime}$ diameter with a gap in its eastern part is seen in the radio maps of Junkes et al. (1992a). They also 
performed $\mathrm{CO}$ observations and suggested a lower distance of $\sim 3 \mathrm{kpc}$ to G 54.4-0.3. IRAS and high resolution CO observations of the surrounding region (Junkes et al. 1992b) show an OB-association, a complex of $\mathrm{H}$ II regions and a $\mathrm{CO}$-shell, all at a distance of $\sim 3 \mathrm{kpc}$. They concluded that G $54.4-0.3$ is associated with these objects and is part of an extended complex of young population I objects, where its progenitor star possibly was born. X-ray emission from G 54.4-0.3 and the surrounding region was detected by ROSAT (Junkes 1996) which is nicely anti-correlated with the cold molecular gas. A hydrogen column density of $10^{22} \mathrm{~cm}^{-2}$ and a plasma temperature of $\sim 2 \times 10^{7} \mathrm{~K}$ were estimated from the thermal X-ray emission.

G 59.8+1.2 was first detected by Reich et al. (1988) in the Effelsberg 2.7-GHz survey, while its radio image was published by Reich et al. (1990). It is classified as a supernova remnant with an incomplete radio shell, an angular size of $\sim 20^{\prime} \times 16^{\prime}$, and a spectral index of $\sim 0.5$ (Green 2004). Radio surveys of the surrounding region, do not reveal any pulsar to be associated with $\mathrm{G} 59.8+1.2$. Neither of the last two remnants has been detected optically in the past.

In this paper, we report the discovery of new faint optical filamentary and diffuse emission from $\mathrm{G}$ 54.4-0.3, G 59.8+1.2 and $\mathrm{G} 126.2+1.6$. We present $\mathrm{H} \alpha+[\mathrm{NII}]$ and in some cases [S II] and [O III] images which reveal filamentary and diffuse structures well correlated with the radio emission. Spectrophotometric observations of the brightest filaments were also performed. In Sect. 2, we present information concerning the observations and data reduction, while the results of the imaging and spectral observations are given in Sects. 3, 4 and 5 for G $126.2+1.6, G 59.8+1.2$ and G 54.4-0.3, respectively. In the last section (Sect. 6) we discuss the physical properties of the supernova remnants.

\section{Observations}

\subsection{Imaging}

The observations were performed with the $0.3 \mathrm{~m}$ SchmidtCassegrain (f/3.2) telescope at Skinakas Observatory in Crete, Greece in July 7, August 27 and 28, September 23 and 27, 2002 and June 25 and 26, 2003. The $1024 \times 1024$ (19 $\mu \mathrm{m}$ pixel) Thomson CCD camera was used resulting in a scale of $4^{\prime \prime}$ pixel $^{-1}$ and a field of view of $70^{\prime} \times 70^{\prime}$.

A series of exposures in $\mathrm{H} \alpha+[\mathrm{N} \mathrm{II}],[\mathrm{O}$ III] and [S II] each of $2400 \mathrm{~s}$ were taken during the observations, resulting in different total exposure times depending on the object. The details of all imaging observations are given in Table 1. The final images in each filter are the average of the individual frames.

The image reduction was carried out using the IRAF and MIDAS packages. The astrometric solution for each field was calculated using reference stars from the Hubble Space Telescope (HST) Guide Star Catalogue (Lasker et al. 1999). The spectrophotometric standard stars HR 5501, HR 7596, HR 7950, and HR 8634 (Hamuy et al. 1992) were used for absolute flux calibration. All coordinates quoted in this work refer to epoch 2000.
Table 1. Imaging and Spectral log.

\begin{tabular}{lccc}
\hline \hline \multicolumn{4}{c}{ Imaging } \\
\hline Object & H $\alpha+[\mathrm{N}$ II $]$ & {$[\mathrm{S} \mathrm{II}]$} & {$[\mathrm{O} \mathrm{III}]$} \\
\hline G 54.4-0.3 & $4800^{a}(2)^{b}$ & $12000(5)$ & $9600(4)$ \\
G 59.8+1.2 & $4800(2)$ & $4800(2)$ & $4800(2)$ \\
G 126.2+1.6 & $4800(2)$ & $9600(4)$ & $9600(4)$ \\
\hline \multicolumn{4}{c}{ Spectroscopy } \\
\hline Area & Slit center & Exp. time $^{a}$ \\
& RA & Dec & No of spectra $^{c}$ ) \\
\hline G 54.4-0.3 (Area Ia) & $19^{\mathrm{h}} 31^{\mathrm{m}} 54^{\mathrm{s}}$ & $19^{\circ} 01^{\prime} 20^{\prime \prime}$ & $3900(1)$ \\
G 54.4-0.3 (Area Ib) & $19^{\mathrm{h}} 31^{\mathrm{m}} 53^{\mathrm{s}}$ & $19^{\circ} 01^{\prime} 13^{\prime \prime}$ & $3900(1)$ \\
G 54.4-0.3 (Area Ic) & $19^{\mathrm{h}} 31^{\mathrm{m}} 53^{\mathrm{s}}$ & $19^{\circ} 01^{\prime} 08^{\prime \prime}$ & $3900(1)$ \\
G 54.4-0.3 (Area II) & $19^{\mathrm{h}} 33^{\mathrm{m}} 06^{\mathrm{s}}$ & $19^{\circ} 13^{\prime} 16^{\prime \prime}$ & $3900(1)$ \\
G 54.4-0.3 (Area III) & $19^{\mathrm{h}} 33^{\mathrm{m}} 14^{\mathrm{s}}$ & $18^{\circ} 38^{\prime} 58^{\prime \prime}$ & $7800(2)$ \\
G 59.8+1.2 & $19^{\mathrm{h}} 38^{\mathrm{m}} 37^{\mathrm{s}}$ & $24^{\circ} 07^{\prime} 45^{\prime \prime}$ & $3900(1)$ \\
G 126.2+1.6 (Area I) & $01^{\mathrm{h}} 17^{\mathrm{m}} 41^{\mathrm{s}}$ & $64^{\circ} 15^{\prime} 58^{\prime \prime}$ & $7200(2)$ \\
G 126.2+1.6 (Area II) & $01^{\mathrm{h}} 17^{\mathrm{m}} 47^{\mathrm{s}}$ & $64^{\circ} 26^{\prime} 32^{\prime \prime}$ & $7800(2)$ \\
\hline
\end{tabular}

${ }^{a}$ Total exposure time in $\mathrm{s}$.

${ }^{b}$ Number of images obtained.

${ }^{c}$ Number of spectra obtained.

\subsection{Spectroscopy}

Low dispersion long-slit spectra were acquired with the $1.3 \mathrm{~m}$ Ritchey-Cretien (f/7.7) telescope at Skinakas Observatory in July 8, August 29, September 12, 2002 and June 29, July 3, 6 and 7,2003 . The 1300 line $\mathrm{mm}^{-1}$ grating was used in conjunction with a $2000 \times 800 \mathrm{SITe} \operatorname{CCD}(15 \mu \mathrm{m}$ pixel $)$ resulting in a scale of $1 \AA$ pixel $^{-1}$ and covers the range of $4750 \AA-6815 \AA$. The spectral resolution is $\sim 8$ pixels and $\sim 11$ pixels full width at half maximum (fwhm) for the red and blue wavelengths, respectively. The slit width is 7. .' 7 and in all cases was oriented in the south-north direction; the slit length is 7.9. Details about the coordinates of the slit centers, the number of spectra and their individual exposure times are given in Table 1. The spectrophotometric standard stars HR 5501, HR 7596, HR 9087, HR 718, and HR 7950 were observed to calibrate the spectra.

\section{The supernova remnant $\mathrm{G} \mathbf{1 2 6 . 2 + 1 . 6}$}

\subsection{The $\mathrm{H} \alpha+[\mathrm{N} I]$, [S II] and [O III] emission line images}

Figure 1 shows the $\mathrm{H} \alpha+[\mathrm{N} \mathrm{II}]$ image, where new faint emission including the known filamentary structures, can be seen. In particular, the image shows several thin and curved filaments all present in the west, south-west and north-west areas of the remnant, while no emission is detected in the east. In Table 2, we list typical fluxes measured in several locations within the field of $\mathrm{G} 126.2+1.6$. The morphology seen in the [S II] filter is similar to that in the $\mathrm{H} \alpha+[\mathrm{N} \mathrm{II}]$ and is not shown here. Both images being flux calibrated provide a first indication of the nature of the observed emission. An examination of the diagnostic ratio $[\mathrm{S} \mathrm{II}] / \mathrm{H} \alpha$ shows that the emission from the brightest part of the remnant originates from shock-heated gas since we estimate ratios $[\mathrm{S} \mathrm{II}] / \mathrm{H} \alpha$ of $0.8-1.2$, which are in agreement with 


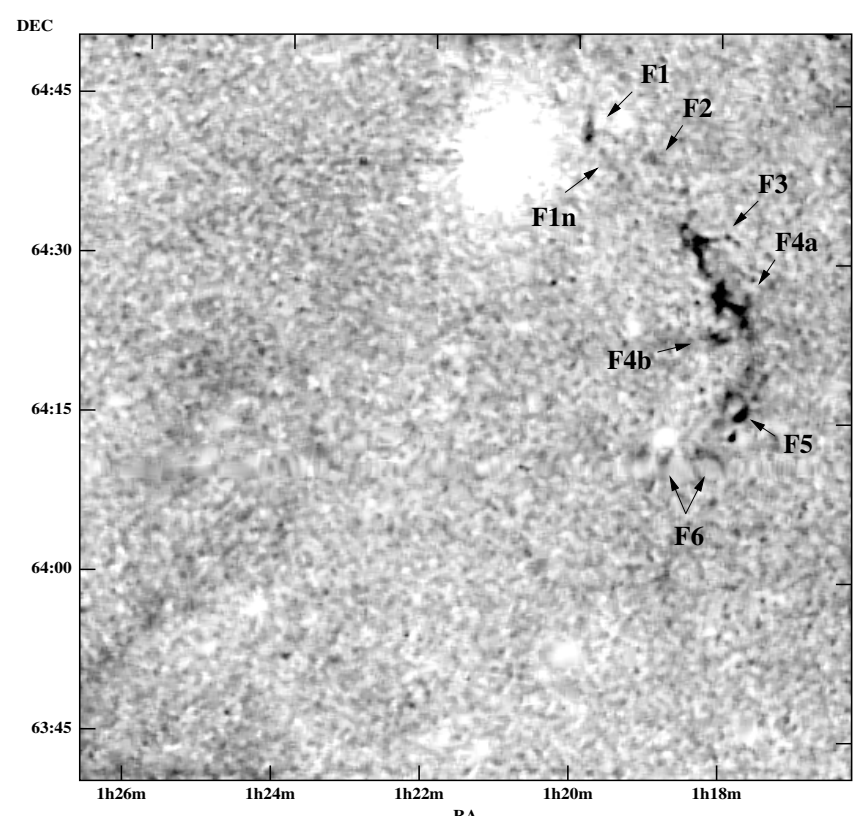

Fig. 1. The field of G $126.2+1.6$ in the $\mathrm{H} \alpha+[\mathrm{N}$ II] filter. Labels F1-F6 define the areas discussed in the text in more detail. Note that F1n defines the area of the new [O III] filament which can be seen clearly in Fig. 2. The image has been smoothed to suppress the residuals from the imperfect continuum subtraction. Shadings run linearly from 0 to $100 \times 10^{-17} \mathrm{erg} \mathrm{s}^{-1} \mathrm{~cm}^{-2} \operatorname{arcsec}^{-2}$.

Table 2. Typically measured fluxes over the brightest filaments.

\begin{tabular}{|c|c|c|c|c|c|c|}
\hline \multicolumn{7}{|c|}{ "G 54.4-0.3 } \\
\hline & $\mathrm{N}$ & $\mathrm{W}(1)$ & $\mathrm{W}(2)$ & $\mathrm{S}$ & & \\
\hline $\mathrm{H} \alpha+[\mathrm{N} \mathrm{II}]$ & 9.5 & 29.6 & 20.0 & 11.0 & & \\
\hline$[\mathrm{S} \mathrm{II}]$ & 1.8 & 6.9 & 5.2 & 1.5 & & \\
\hline [O III] & & & & $7.3^{c}$ & & \\
\hline \multicolumn{7}{|c|}{ G 59.8+1.2 } \\
\hline & $\mathrm{W}$ & SW & SE & $\mathrm{NE}(\mathrm{I})^{a}$ & $\mathrm{NE}(\mathrm{O})^{a}$ & $\mathrm{E}^{b}$ \\
\hline $\mathrm{H} \alpha+[\mathrm{N} \mathrm{II}]$ & 21.8 & 28.0 & 18.3 & 15.2 & 17.2 & 32.5 \\
\hline$[\mathrm{S} \mathrm{II}]$ & 3.8 & 5.9 & 2.4 & 5.2 & 11.8 & 5.8 \\
\hline [O III] & \multicolumn{6}{|c|}{$<8.5^{c}$} \\
\hline \multicolumn{7}{|c|}{ G 126.2+1.6 } \\
\hline & $\mathrm{N}$ & $\mathrm{NW}$ & $\mathrm{W}$ & SW & $S$ & \\
\hline $\mathrm{H} \alpha+[\mathrm{N}$ II $]$ & 14.2 & 17.7 & 26.3 & 29.0 & 5.8 & \\
\hline [S II $]$ & 4.8 & 7.0 & 8.2 & 8.5 & 2.6 & \\
\hline [O III] & 6.9 & 5.8 & 8.8 & 19.1 & 2.8 & \\
\hline
\end{tabular}

Fluxes in units of $10^{-17} \mathrm{erg} \mathrm{s}^{-1} \mathrm{~cm}^{-2} \operatorname{arcsec}^{-2}$

Median values over a $40^{\prime \prime} \times 40^{\prime \prime}$ box.

${ }^{a}$ Inner (I) and outer (O) filaments as defined in Sect. 4.1.

${ }^{b}$ The unknown east bright region outside the snr's borders.

${ }^{c} 3 \sigma$ upper limit.

our spectra measurements (Sect. 3.3). Each filter contribution was estimated using the methodology of Mavromatakis et al. (2002b).

Morphologically, starting from the north area, a bright filament 2' long (named F1 in Fig. 1) is present which lies a few arcminutes to the west of the variable star HD 8003 with its center approximately at $\alpha \simeq 1^{\mathrm{h}} 19^{\mathrm{m}} 51^{\mathrm{s}}$ and $\delta \simeq 64^{\circ} 42^{\prime} 13^{\prime \prime}$. South-west of this filament appears a much fainter one (F2)

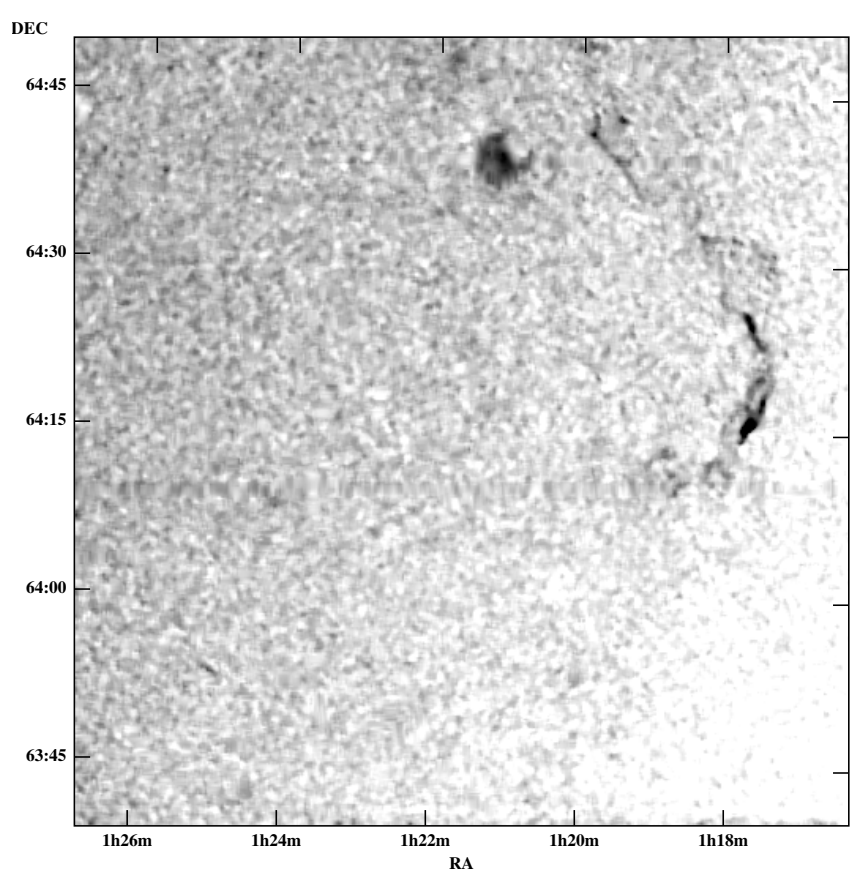

Fig. 2. The field of $\mathrm{G} 126.2+1.6$ in the [O III] filter. The image has been smoothed to suppress the residuals from the imperfect continuum subtraction. Shadings run linearly from 0 to $18 \times$ $10^{-17} \mathrm{erg} \mathrm{s}^{-1} \mathrm{~cm}^{-2} \operatorname{arcsec}^{-2}$.

at $\alpha \simeq 1^{\mathrm{h}} 18^{\mathrm{m}} 58^{\mathrm{s}}, \delta \simeq 64^{\circ} 40^{\prime} 00^{\prime \prime}$ which is up to $1^{\prime}$ long. Further to the south-west, a prominent new bright structure which appears exactly to the north of the known optical filament in the west (Rosado 1982). This structure (named F3, $7^{\prime}$ long, $\sim 1$ '.2 wide) lies between $\alpha \simeq 1^{\mathrm{h}} 18^{\mathrm{m}} 22^{\mathrm{s}}, \delta \simeq 64^{\circ} 33^{\prime} 10^{\prime \prime}$ and $\alpha \simeq$ $1^{\mathrm{h}} 18^{\mathrm{m}} 08^{\mathrm{s}}, \delta \simeq 64^{\circ} 28^{\prime} 41^{\prime \prime}$ has the same curvature and separated by a $\sim 2^{\prime}$ gap with the known west filament. A much more detailed image of the known optical emission can be seen in Fig. 1. The west structure consist of two main parts centered at $\alpha \simeq 1^{\mathrm{h}} 17^{\mathrm{m}} 48^{\mathrm{s}}, \delta \simeq 64^{\circ} 25^{\prime} 58^{\prime \prime}(\mathrm{F} 4 \mathrm{a})$ and $\alpha \simeq 1^{\mathrm{h}} 18^{\mathrm{m}} 02^{\mathrm{s}}, \delta \simeq$ $64^{\circ} 22^{\prime} 53^{\prime \prime}$ (F4b) and separated by a few arcminutes. Diffuse emission is also present to the east and south of these structures. Another $\sim 8^{\prime}$ long filament (F5) which appears to the south has also the same curvature with the west filament and lies between $\alpha \simeq 1^{\mathrm{h}} 17^{\mathrm{m}} 37^{\mathrm{s}}, \delta \simeq 64^{\circ} 16^{\prime} 55^{\prime \prime}$ and $\alpha \simeq 1^{\mathrm{h}} 17^{\mathrm{m}} 50^{\mathrm{s}}$, $\delta \simeq 64^{\circ} 15^{\prime} 14^{\prime \prime}$. Diffuse emission appears along north and south of this filament. Finally, there are extremely faint new filaments (Area F6, 2-3') in the area between $\alpha \simeq 1^{\mathrm{h}} 17^{\mathrm{m}} 58^{\mathrm{s}}-1^{\mathrm{h}} 19^{\mathrm{m}} 06^{\mathrm{s}}$ and $\delta \simeq 64^{\circ} 08^{\prime}-64^{\circ} 12^{\prime}$ which also seem to have the same curvature with the other filaments.

\subsection{The $[\mathrm{O} I I I]$ emission line image}

The detected [O III] emission (Fig. 2) appears more filamentary and less diffuse than in the $\mathrm{H} \alpha+[\mathrm{N} \mathrm{II}]$ image. In Table 2 typical [O III] fluxes are listed. Significant differences between the $\mathrm{H} \alpha+[\mathrm{N} \mathrm{II}]$ and $[\mathrm{O}$ III] images are present for most of the filaments. In particular, to the north in contrast to the $2^{\prime}$ filament (F1) found in $\mathrm{H} \alpha+[\mathrm{N}$ II $]$, there is a new faint thin filament (F1n $-13^{\prime}$ long) which lies between $\alpha \simeq 1^{\mathrm{h}} 19^{\mathrm{m}} 56^{\mathrm{s}}$, $\delta \simeq 64^{\circ} 42^{\prime} 15^{\prime \prime}$ and $\alpha \simeq 1^{\mathrm{h}} 19^{\mathrm{m}} 11^{\mathrm{s}}, \delta \simeq 64^{\circ} 35^{\prime} 58^{\prime \prime}$ and 
Table 3. Relative line fluxes.

\begin{tabular}{lllllllll}
\hline \hline & \multicolumn{3}{c}{ G 54.4-0.3 } & \multicolumn{3}{c}{ G 59.8+1.2 } & \multicolumn{2}{c}{ G 126.2+1.6 } \\
& Area Ia & Area Ib & Area Ic & Area II & & Area Ia $^{1}$ & Area Ib $^{1}$ & Area II \\
Line $(\AA)$ & $F^{2,3}$ & $F^{2,3}$ & $F^{2,3}$ & $F^{2,3}$ & $F^{2,3}$ & $F^{2,3}$ & $F^{2,3}$ & $F^{2,3}$ \\
\hline $4861 \mathrm{H} \beta$ & $<6$ & $<5$ & $<3$ & $<3$ & $<8$ & $<6$ & $12(3)$ & $6(2)$ \\
$4959[\mathrm{O}$ III] & - & - & - & - & - & $87(15)$ & $114(16)$ & $<6$ \\
$5007[\mathrm{O} \mathrm{III}]$ & - & - & - & - & - & $286(39)$ & $372(35)$ & $26(10)$ \\
$6548[\mathrm{~N} \mathrm{II}]$ & $9(3)$ & $18(10)$ & $12(10)$ & $26(6)$ & $18(20)$ & $24(6)$ & $23(3)$ & $17(12)$ \\
$6563 \mathrm{H} \alpha$ & $100(29)$ & $100(57)$ & $100(64)$ & $100(19)$ & $100(84)$ & $100(117)$ & $100(15)$ & $100(70)$ \\
$6584[\mathrm{~N} \mathrm{II}]$ & $35(10)$ & $53(30)$ & $35(27)$ & $73(14)$ & $51(46)$ & $114(31)$ & $107(21)$ & $61(42)$ \\
$6716[\mathrm{~S} \mathrm{II}]$ & $34(10)$ & $40(27)$ & $35(27)$ & $31(6)$ & $35(32)$ & $52(12)$ & $56(10)$ & $54(37)$ \\
$6731[\mathrm{~S} \mathrm{II}]$ & $26(8)$ & $26(19)$ & $24(19)$ & $30(6)$ & $33(31)$ & $46(18)$ & $50(14)$ & $39(26)$ \\
\hline $\mathrm{Absolute} \mathrm{H} \alpha$ flux & 45.2 & 6.7 & 10.1 & 3.0 & 3.2 & 6.7 & 6.5 & 16.8 \\
$\mathrm{H} \alpha / \mathrm{H} \beta$ & $>17$ & $>20$ & $>30$ & $>28$ & $>12$ & $>8$ & 8.3 & 16.7 \\
{$[\mathrm{~S} \mathrm{II}] / \mathrm{H} \alpha$} & $0.60(14)$ & $0.67(28)$ & $0.60(29)$ & $0.60(8)$ & $0.68(39)$ & $0.98(22)$ & $1.06(12)$ & $0.93(44)$ \\
$\mathrm{F}(6716) / \mathrm{F}(6731)$ & $1.34(7)$ & $1.54(15)$ & $1.48(15)$ & $1.03(5)$ & $1.06(22)$ & $1.13(10)$ & $1.12(8)$ & $1.37(22)$ \\
{$[\mathrm{O} \mathrm{III}] / \mathrm{H} \beta$} & - & - & - & - & - & $>30$ & 32.4 & 5.3 \\
$\mathrm{c}(\mathrm{H} \beta)^{5}$ & $>2.20$ & $>2.43$ & $>3.06$ & $>3.06$ & $>1.85$ & $>1.85$ & $1.34(3)$ & $2.21(2)$ \\
\hline
\end{tabular}

${ }^{1}$ Areas Ia and $\mathrm{Ib}$, having an offset of $8^{\prime \prime}$ and 9 " south and north of the slit center, respectively.

${ }^{2}$ Listed fluxes are a signal to noise weighted average of two fluxes.

${ }^{3}$ Fluxes normalized to $F(\mathrm{H} \alpha)=100$ and are uncorrected for interstellar extinction.

${ }^{4}$ In units of $10^{-17} \mathrm{erg} \mathrm{s}^{-1} \mathrm{~cm}^{-2} \operatorname{arcsec}^{-2}$.

${ }^{5}$ The logarithmic extinction is derived by $c=1 / 0.348 \times \log \left((\mathrm{H} \alpha / \mathrm{H} \beta)_{\mathrm{obs}} / 2.85\right)$.

Numbers in parentheses represent the signal to noise ratio of the quoted fluxes.

follows the same curvature with the west filament. This new long filament is also very well correlated with the $4850 \mathrm{MHz}$ radio map (Fig. 3). On the other hand, the new $7^{\prime}$ bright $\mathrm{H} \alpha+[\mathrm{N} \mathrm{II}]$ filament (F3) does not have [O III] counterpart and at the same location only very faint diffuse emission is found. The known west structure F4 (detected in [O III] by Fesen et al. 1983) appears also bright but thinner in [O III] than $\mathrm{H} \alpha+[\mathrm{N} \mathrm{II}]$. The 8 ' filament F5 (detected in [O III] by Blair et al. 1980; Fesen et al. 1983) displays a different morphology between the lower ionization lines of $\mathrm{H} \alpha+[\mathrm{N} \mathrm{II}]$ and [S II] and the medium ionization line of $[\mathrm{O} \mathrm{III]}$ where it is much brighter and better defined. Finally, A similar situation appears both in [O III] and $\mathrm{H} \alpha+[\mathrm{N} \mathrm{II}]$ in the areas where weak and diffuse emission is found.

\subsection{The optical spectra from $G 126.2+1.6$}

The deep low resolution spectra were taken on the relatively bright optical filaments at two different locations (Table 1). In Table 3, we quote the relative line fluxes taken from the above locations (designated Area I and II). In particular, in Area I, we extracted two different apertures (Ia and Ib) along the slit that are free of field stars and include sufficient line emission to allow an accurate determination of the observed lines. The background extraction apertures were selected towards the north and south ends of each slit depending on the filament's position within the slit. The measured line fluxes indicate emission from shock heated gas, since $[\mathrm{S} \mathrm{II}] / \mathrm{H} \alpha \simeq 1.0$. The spectra indicate significant attenuation of the optical radiation but given the low counting statistics we cannot attribute the extinction variations to intrinsic absorption of the object. The signal to

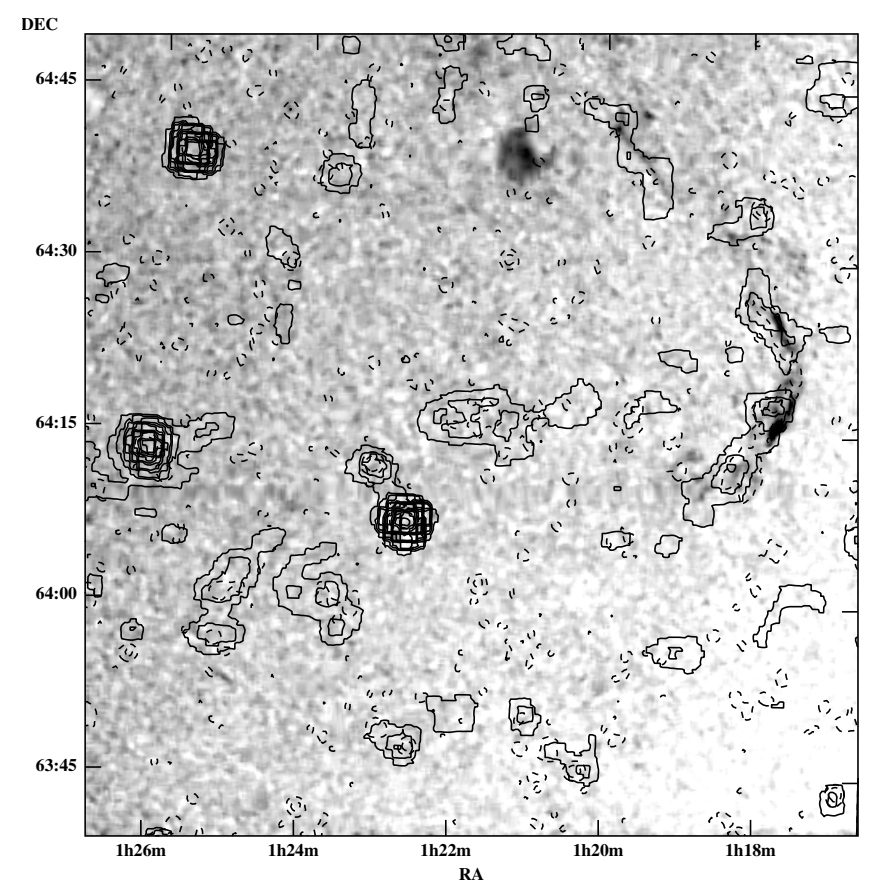

Fig. 3. The correlation between the [O III] emission and the radio emission at $1400 \mathrm{MHz}$ (dashed line) and $4850 \mathrm{MHz}$ (solid line) for G $126.2+1.6$ is shown in this figure. The $1400 \mathrm{MHz}$ and the $4850 \mathrm{MHz}$ radio contours scale linearly from $1.1 \times 10^{-3}$ to $0.01 \mathrm{Jy} /$ beam (resolution $\left.45^{\prime \prime} \times 45^{\prime \prime}\right)$ and $7 \times 10^{-3}$ to $0.1 \mathrm{Jy} /$ beam $\left(7^{\prime} \times 7^{\prime}\right)$, respectively.

noise ratios do not include calibration errors, which are less than 10 percent.

The absolute $\mathrm{H} \alpha$ flux covers a range of values, from 6.5 to $16.8 \times 10^{-17} \mathrm{erg} \mathrm{s}^{-1} \mathrm{~cm}^{-2} \operatorname{arcsec}^{-2}$. The [S II] $\lambda \lambda 6716 / 6731$ ratio which was calculated between 1.1 and 1.4 , indicates 
electron densities between 30 to $400 \mathrm{~cm}^{-3}$ (Osterbrock 1989). However, taking into account the statistical errors on the sulfur lines, we calculate that electron densities up to $600 \mathrm{~cm}^{-3}$ are allowed (Shaw \& Dufour 1995). Furthermore, as noted above, $\mathrm{H} \beta$ emission was detected in the spectra of Area Ib and II while in filament of Area Ia only an upper limit is given. However, measurements for the $[\mathrm{O} \mathrm{III}] / \mathrm{H} \beta$ ratio result in values larger than 30 (Area I) and less than 6 (Area II). Theoretical models of Cox \& Raymond (1985) and Hartigan et al. (1987) suggest that for shocks with complete recombination zones this value is $\sim 6$, while this limit is exceeded in case of shock with incomplete recombination zones (Raymond et al. 1988). Our measured values suggest, in Area I that shocks with incomplete recombination zones are present, while in Area II shocks with complete recombination zones. Therefore, according to our measurements and the above theoretical models the estimated shock velocities are $\sim 100 \mathrm{~km} \mathrm{~s}^{-1}$ and $\sim 120 \mathrm{~km} \mathrm{~s}^{-1}$ for Area II and I, respectively.

\subsection{Observations at other wavelengths}

Several radio observations of $\mathrm{G} 126.2+1.6$ have been performed in the past (see Sect. 1 for detail). The optical filaments match very well the radio emission of G 126.2+1.6 at $1410 \mathrm{MHz}$ (Reich et al. 1979) and $4850 \mathrm{MHz}$ (Fürst et al. 1984), suggesting their association (Fig. 3). Note that a first indication of such correlation was made by Blair et al. (1980) who drawn a sketch locating their optical [O III] filaments with respect to the radio contours at $1400 \mathrm{MHz}$. The area of $\mathrm{G} 126.2+1.6$ was observed by ROSAT during the All-Sky survey but no emission was detected. A small part of the remnant was in the field of view of a ROSAT pointed observation (RP400291N00). The image of this observation reveals extended emission $\left(\sim 7.5 \times 15^{\prime}\right)$ centered at $1^{\mathrm{h}} 21^{\mathrm{m}} 52^{\mathrm{s}}, 64^{\circ} 32^{\prime} 50^{\prime \prime}$. The typical flux of this structure is $\sim 1.1 \times 10^{-3}$ cts s$^{-1}$ arcmin $^{-2}$ and seems to be present both in the $0.5-1.0 \mathrm{keV}$ and the $1.0-2.4 \mathrm{keV}$ bands. However, it may be difficult to correlate this emission to $\mathrm{G} 126.2+1.6$ because it is located close to the edge of the field of view of the PSPC $\left(\sim 51^{\prime}\right.$ from the center). The IRAS maps have also been searched at $12,25,60$ and $100 \mu \mathrm{m}$ but no features which might suggest an interaction of the supernova remnant with an IR source were found.

\section{The supernova remnant $G 59.8+1.2$}

\subsection{The $H \alpha+[N I I,[S I I]$ and [O III] emission line images}

Optical filamentary and diffuse emission is detected for a first time for this remnant. The major characteristic revealed from the $\mathrm{H} \alpha+[\mathrm{NII}]$ image (Fig. 4) seems to be the low surface brightness of $\mathrm{G} 59.8+1.2$. Weak, diffuse emission is present in the south, south-east and central areas of the remnant, while no emission is detected in the north. The most interesting region lies in the west, where a complex filamentary structure exists (between $\alpha \simeq 19^{\mathrm{h}} 38^{\mathrm{m}} 30.7^{\mathrm{s}}, \delta \simeq 24^{\circ} 10^{\prime} 03^{\prime \prime}$ and $\alpha \simeq$ $\left.19^{\mathrm{h}} 38^{\mathrm{m}} 08.2^{\mathrm{s}}, \delta \simeq 24^{\circ} 15^{\prime} 38^{\prime \prime}\right)$, which is very well correlated

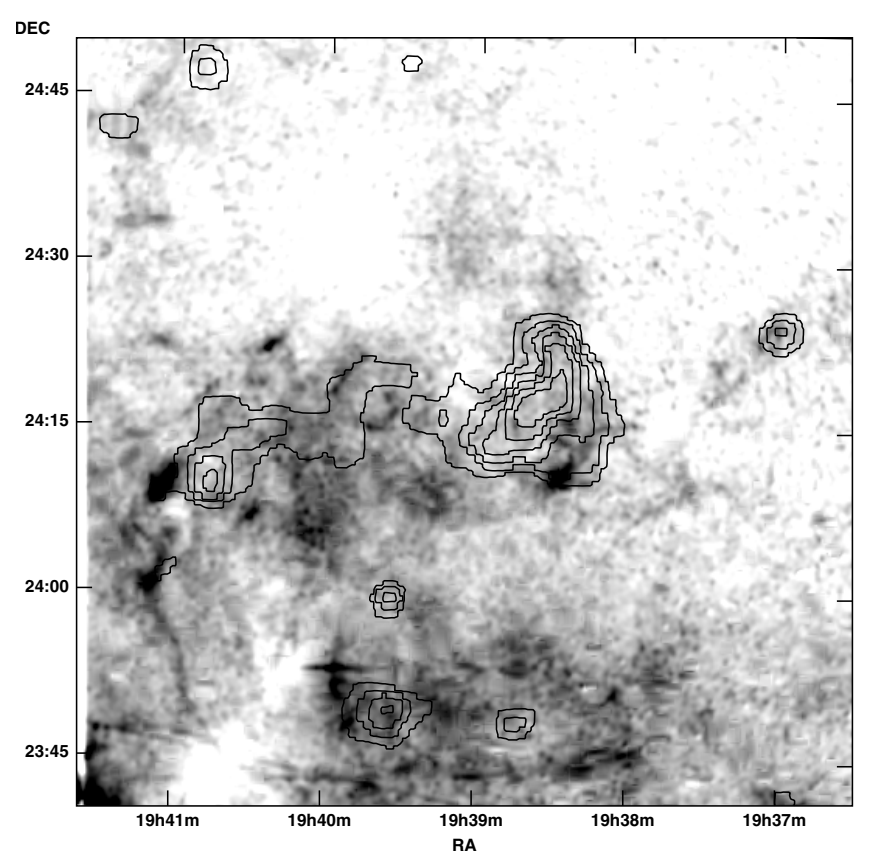

Fig. 4. The correlation between the $\mathrm{H} \alpha+[\mathrm{N}$ II] emission of $\mathrm{G} 59.8+1.2$ and the radio emission at $4850 \mathrm{MHz}$ (solid line) is shown in this figure. The image has been smoothed to suppress the residuals from the continuum subtraction. Shadings run linearly from 0 to $120 \times$ $10^{-17} \mathrm{erg} \mathrm{s}^{-1} \mathrm{~cm}^{-2} \operatorname{arcsec}^{-2}$. The $4850 \mathrm{MHz}$ radio contours scale linearly from $2.0 \times 10^{-2} \mathrm{Jy} /$ beam to $0.15 \mathrm{Jy} /$ beam.

with the radio emission. This bright filament extends for $\sim 8^{\prime}$ in the west, south-west. In Table 2, we present typical average fluxes measured in several locations within the field of G 59.8+1.2 including an unknown $\mathrm{H}$ II region which is located south and south-east of the remnant. In contrast to the previous results, diffuse emission was mainly detected in the [S II] emission line image, while no significant emission was found in the image of the [O III] medium ionization line. We did not detect $[\mathrm{S} \mathrm{II}]$ emission where most of the $\mathrm{H} \alpha+[\mathrm{N} \mathrm{II}]$ emission was found but only where the bright west filament appears. Therefore, the [S II] and [O III] images are not shown here. Table 2 lists typical [S II] and [O III] (3- $\sigma$ upper limits) fluxes measured in different parts of the remnant. A deeper study of these images shows that the emission from the brightest part of the remnant (west filament) originates from shock heated gas since we estimate a ratio $[\mathrm{S} \mathrm{II]}] / \mathrm{H} \alpha \sim 0.4-0.6$, while a photoionization mechanism acts in the south-east region $([\mathrm{S} \mathrm{II}] / \mathrm{H} \alpha \sim 0.2-0.3)$.

Assuming that the east filaments belong to the remnant, their geometry allows us to approximately define its diameter. In particular, two possibilities will be examined. If the east border of the remnant is defined by the outer filament at $\alpha \simeq$ $19^{\mathrm{h}} 39^{\mathrm{m}} 53.0^{\mathrm{s}}, \delta \simeq 24^{\circ} 19^{\prime} 51^{\prime \prime}$, then a diameter of $22^{\prime} \times 20^{\prime} .5$ can be derived with its center at $\alpha \simeq 19^{\mathrm{h}} 39^{\mathrm{m}} 04^{\mathrm{s}}, \delta \simeq 24^{\circ} 17^{\prime} 15^{\prime \prime}$, while if the inner filament at $\alpha \simeq 19^{\mathrm{h}} 39^{\mathrm{m}} 31.0^{\mathrm{s}}, \delta \simeq 24^{\circ} 15^{\prime} 35^{\prime \prime}$ defines the remnant's east border then its diameter is $19^{\prime} \times 16^{\prime}$ with the centre now to be at $\alpha \simeq 19^{\mathrm{h}} 39^{\mathrm{m}} 00^{\mathrm{s}}, \delta \simeq 24^{\circ} 14^{\prime} 18^{\prime \prime}$. Note that the latter optical angular size is in very good agreement with the value of $\sim 20^{\prime} \times 16^{\prime}$ given in Green's catalogue (Green 2004). However, the larger angular diameter cannot be 
excluded since the infrared emission (Sect. 4.3) is in agreement with the larger diameter value, X-ray emission has not been detected so far and the radio shell is incomplete.

\subsection{The spectra of $G 59.8+1.2$}

A low resolution spectrum was taken at a bright area (see Table 1 for position details) among the diffuse emission seen in the $\mathrm{H} \alpha+[\mathrm{N} \mathrm{II}]$ image, which coincides with the non-thermal radio emission. The selected aperture and background are chosen using the same criteria as for G 126.2+1.6 (Sect. 3.3). The measured fluxes are given in Table 3 . The $[\mathrm{S} \mathrm{II}] / \mathrm{H} \alpha$ ratio of 0.68 shows that the optical radiation originates from shocked gas. [O III] emission was not detected while the very weak $\mathrm{H} \beta$ emission suggests significant interstellar attenuation of the optical emission. Using the latter emission, the upper limit of the interstellar extinction $\mathrm{c}(\mathrm{H} \beta)$ is 1.85 . The absence of [O III] emission can be explained by slow shocks $\left(\leq 70 \mathrm{~km} \mathrm{~s}^{-1}\right)$ since the presence of higher velocities shocks would produce detectable [O III] emission. Furthermore, the $\mathrm{H} \beta$ emission upper limit and the absence of $[\mathrm{OIII}]$, result in values of the $[\mathrm{O} \mathrm{III}] / \mathrm{H} \beta$ ratio of less than 1 . This suggests that shocks with complete recombination zones are likely to be present. The absolute $\mathrm{H} \alpha$ flux was measured to be $3.2 \times 10^{-17} \mathrm{erg} \mathrm{s}^{-1} \mathrm{~cm}^{-2} \operatorname{arcsec}^{-2}$. The [S II] $\lambda \lambda$ 6716/6731 ratio which was calculated to 1.06 , indicates electron densities to $470 \mathrm{~cm}^{-3}$ according to the task "temden" in the nebular package in IRAF (Shaw \& Dufour 1995). However, taking into account the statistical errors on the sulfur lines, we calculate that electron densities as low as $380 \mathrm{~cm}^{-3}$ and as high as $580 \mathrm{~cm}^{-3}$ are allowed.

\subsection{Observations at other wavelengths}

The optical emission matches very well the radio emission of G 59.8+1.2 at $4850 \mathrm{MHz}$, suggesting their correlation (Fig. 4). The observed filament is located close to the outer edge of the radio contours but the low resolution of the radio images does not allow us to determine the relative position of the filament with respect to the shock front. In order to explore how the infrared emission correlates with the optical emission, IRAS resolution-enhanced (HiRes; Aumann et al. 1990) images at $60 \mu \mathrm{m}$ of the same area were examined. Figure 5 shows a deep greyscale representation of the optical emission shown in Fig. 4 with overlapping contours of the infrared emission $(60 \mu \mathrm{m})$. IR emission correlated with G $59.8+1.2$ is found towards the south and west. The bright emission in the south-east coincides with the optical emission therefore an association cannot be ruled out, and especially in the case of a larger diameter remnant (Sect. 4.1). Finally, X-ray emission was not detected in the ROSAT All-sky survey.

\section{The supernova remnant G 54.4-0.3}

\subsection{The $\mathrm{H} \alpha+[\mathrm{N} I I],[S I I]$ and [O III] emission line images}

Optical emission from $\mathrm{G}$ 54.4-0.3 is detected for the first time as for $\mathrm{G} 59.8+1.2$. The $\mathrm{H} \alpha+[\mathrm{N} \mathrm{II}]$ image (Fig. 6) shows

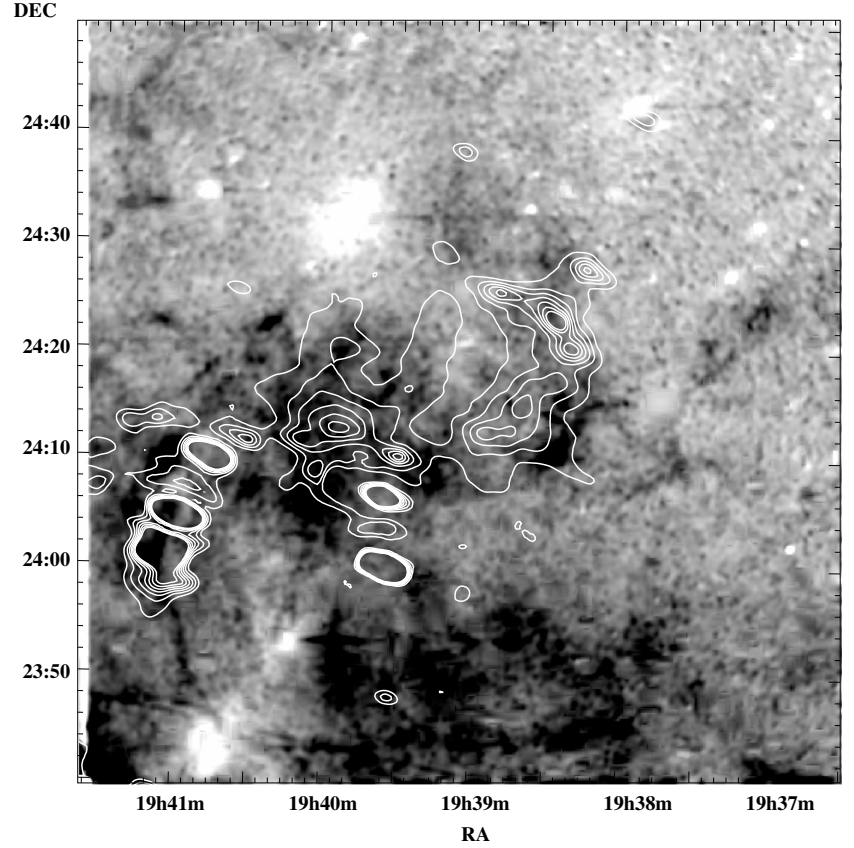

Fig. 5. The correlation between the deep negative greyscale representation of the $\mathrm{H} \alpha+[\mathrm{N} \mathrm{II}]$ emission and the IRAS $60 \mu \mathrm{m}$ (HiRes, white line) is shown in this figure. The $\mathrm{H} \alpha+[\mathrm{N} \mathrm{II}]$ image and the $60 \mu \mathrm{m}$ contours scale linearly from 0 to $90 \times 10^{-17} \mathrm{erg} \mathrm{s}^{-1} \mathrm{~cm}^{-2} \operatorname{arcsec}^{-2}$ and from 8 to $40 \mathrm{MJy} / \mathrm{sr}$, respectively.

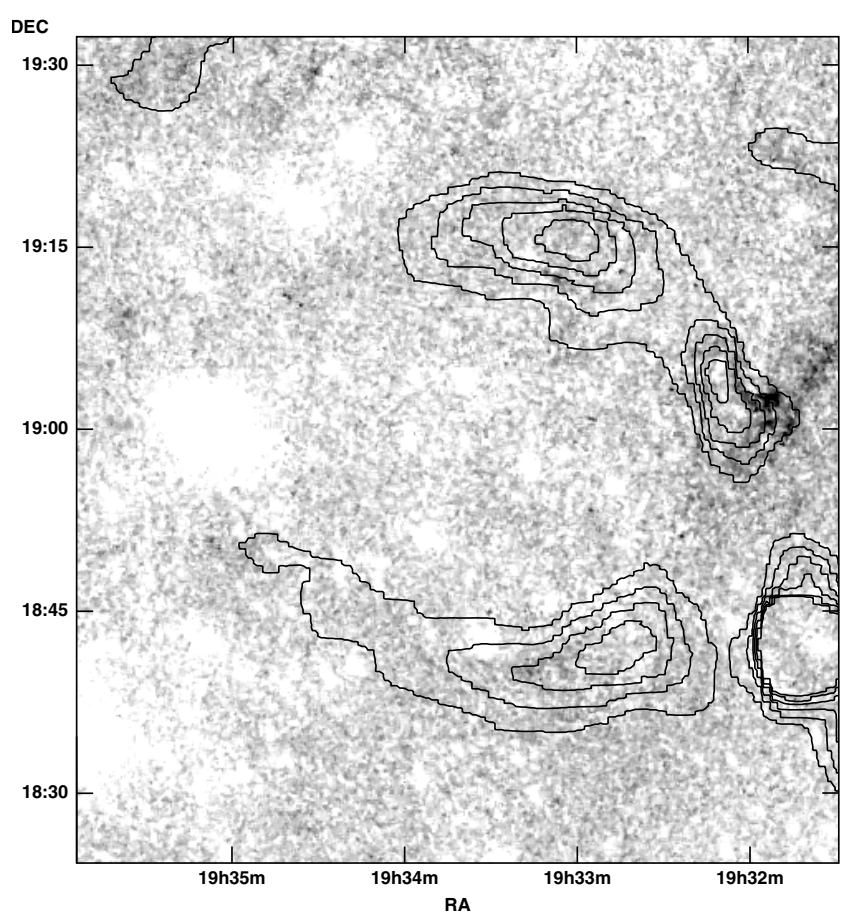

Fig. 6. As Fig. 4 but for G 54.4-0.3. Shadings run linearly from 0 to $50 \times 10^{-17} \mathrm{erg} \mathrm{s}^{-1} \mathrm{~cm}^{-2} \operatorname{arcsec}^{-2}$. The $4850 \mathrm{MHz}$ radio contours scale linearly from $3.5 \times 10^{-2} \mathrm{Jy} /$ beam to $0.25 \mathrm{Jy} /$ beam.

filamentary but also diffuse emission mainly in the west edge. The morphology of the [S II] image is generally similar to, though not as bright as, that of the $\mathrm{H} \alpha+[\mathrm{N}$ II] image and is not shown here, while no significant [O III] emission was found. The flux calibrated images of $\mathrm{H} \alpha+[\mathrm{N}$ II] and [S II] provide 


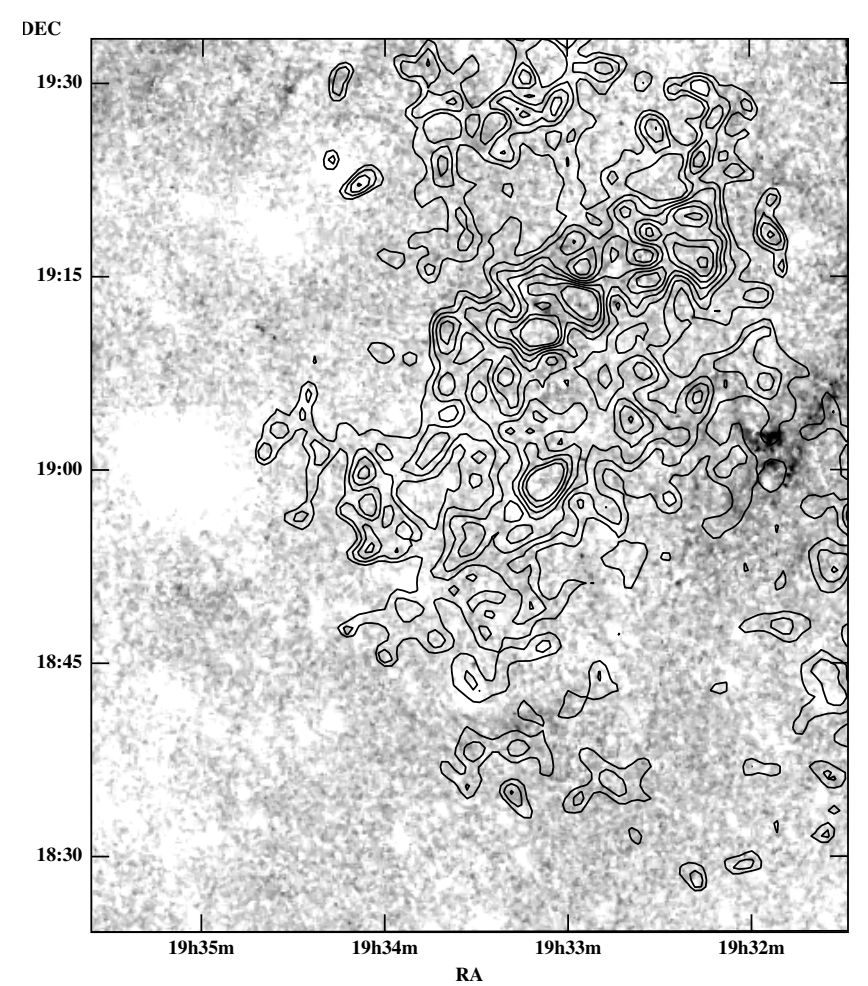

Fig. 7. As Fig. 6 but with the X-ray ASCA at $0.5-10 \mathrm{keV}$ (contour) emission of G 54.4-0.3. The X-ray contours scale linearly from $2.1 \times$ $10^{-4} \mathrm{Jy} /$ beam to $3.5 \times 10^{-4} \mathrm{Jy} /$ beam.

a first indication to the nature of the observed emission (see Table 2). A study in different parts shows that the emission from the west area originates from shock heated gas since ratios $[\mathrm{S} \mathrm{II}] / \mathrm{H} \alpha$ of $\sim 0.6-0.7$ were found, while measurements of very faint emission to the north and south of the remnant's edge show a ratio $[\mathrm{S} \mathrm{II}] / \mathrm{H} \alpha$ of $\sim 0.4-0.5$ and $\sim 0.3-0.4$, respectively. It is possible that a photoionization mechanism acts in these areas.

\subsection{The long-slit spectra from $G 54.4-0.3$}

The low resolution spectra cover the west (Area Ia-c), north (Area II) and south (Area III) parts of the remnant (their exact positions are given in Table 1). Spectra from Areas I \& II suggest that the detected emission originates from shock heated gas ([S II $] / \mathrm{H} \alpha \sim 0.6)$, while no significant emission was detected in Area III. In general, the spectra of G 54.4-0.3 show similar results with that of $\mathrm{G} 59.8-1.2$ (Sect. 4.2) concerning the appearance of the $\mathrm{H} \beta$ and $[\mathrm{O} \mathrm{III}]$ emission lines, hence the presence of slow shocks, strong interstellar extinction and complete recombination zones can be attributed. The absolute $\mathrm{H} \alpha$ flux covers a range of values from 3.0 to $15.2 \times$ $10^{-17} \mathrm{erg} \mathrm{s}^{-1} \mathrm{~cm}^{-2} \operatorname{arcsec}^{-2}$, while the [S II] $\lambda \lambda$ 6716/6731 ratio of 1.03 (Area II) and 1.43 (mean of Area Ia-c) indicate low electron densities (up to $\approx 500 \mathrm{~cm}^{-3}$ ).

\subsection{Observations at other wavelengths}

The newly discovered optical emission appears correlated with the radio map of G 54.4-0.3 at $4850 \mathrm{MHz}$ (Fig. 6).
The IRAS (HiRes) images at $60 \mu \mathrm{m}$ were also searched for emission features that could be correlated with the optical ones. The detected IR emission may not actually be in the area of the remnant but could be part of a complex region (OB-association, H II regions, CO-shell; Junkes et al. 1992b). Archival ASCA data (Gotthelf 2001) have been used in order to investigate further the remnant's physical properties. G 54.4-0.3 was observed by the ASCA satellite on October 14-16, 1994 with the GIS3 detector covering the energy band of $0.5-10 \mathrm{keV}$. Three sets of ASCA data pointed toward the centre, north and southwest portion of the remnant were jointed to create the final mosaic (Fig. 7). Diffuse X-ray emission is present in the area of this remnant correlates well with the optical emission found to the west.

\section{Discussion}

The supernova remnants $G 59.8+1.2$ and $G$ 54.4-0.4 are among the least observed remnants in optical wavelengths while the G 126.2+1.6 new optical data improve the knowledge we had so far concerning its physical properties.

\subsection{G 126.2+1.6}

This remnant shows up as an incomplete circular shell in optical and radio wavelengths. Faint diffuse X-ray emission is also detected in the area but its relation to $G 126.2+1.6$ is not clear. Our images show the known emission and reveal more new filamentary and diffuse structures. The new filament detected in the north, north-west is very well correlated with the radio emission. The calibrated images as well as the long-slit spectra suggest that the detected emission results from shock-heated gas. This remnant may belong to the group of remnants which display partially strong [O III] emission like e.g. CTB1 (Fesen et al. 1997), G 114.3+0.3 (Mavromatakis et al. 2002a), G 17.4-2.3 (Boumis et al. 2002). The morphological differences between the low and medium ionization lines (Sects. 3.1, 3.2) suggest the presence of significant inhomogeneities and density variations in the preshock medium (Hester 1987). Despite the low emission detected at $\mathrm{H} \beta$, an estimation of the $[\mathrm{O} \mathrm{III}] / \mathrm{H} \beta$ ratio suggest values greater than 30 (Area I) and less than 6 (Area II). Large $[\mathrm{O}$ III $] / \mathrm{H} \beta$ ratios are not unusual, since they have been measured by Fesen et al. (1983) and Mavromatakis et al. (2002b) in G 65.3+5.7 indicating shocks with incomplete recombination zones to be present. The observed variation in the absolute line fluxes could be due to variations of the interstellar cloud densities, shock velocities or intrinsic absorption.

A hydrogen column density $N_{\mathrm{H}}$ between 7.8 and $9.4 \times$ $10^{21} \mathrm{~cm}^{-2}$ is given by Dickey \& Lockman (1990) in the direction of the optical filaments. Using the statistical relation of Predehl \& Schmitt (1995), we obtain an $N_{\mathrm{H}}$ of $5.0 \times$ $10^{21} \mathrm{~cm}^{-2}$ and $8.3 \times 10^{21} \mathrm{~cm}^{-2}$ for the minimum and maximum c values calculated from our spectra (Table 3 ), respectively. Furthermore, using the $E_{B-V}$ value adopted from the SFD code (Schlegel et al. 1998) an $N_{\mathrm{H}}$ of $7.9 \times 10^{21} \mathrm{~cm}^{-2}$ was calculated. Both values (our spectra and SFD) are consistent with the estimated galactic $N_{\mathrm{H}}$ considering the uncertainties involved. 
Joncas et al. (1989) argue that the distance to G $126.2+1.6$ is most likely to lie in the range 2 to $5 \mathrm{kpc}$. Blair et al. (1980) suggested that a missing hole of $\mathrm{HI}$ lying at $2.4 \pm 0.4 \mathrm{kpc}$ could correspond to the ionized volume of $\mathrm{G} 126.2+1.6$. Following Dopita (1979), a lower limit to the preshock density (magnetic fields not included) can be estimated as $n_{0}>13.3 \mathrm{~cm}^{-3}$ and the energy of the SN explosion is then $E_{51}>0.26 D_{\mathrm{kpc}}^{3} \sim$ $7 \times 10^{51} \mathrm{erg}$. Inputting all of these results into a standard Sedov model (see, e.g., the Appendix of Claas et al. 1989) yields the shock speed of $\sim 100 \mathrm{~km} \mathrm{~s}^{-1}$ and postshock temperature of $5 \times 10^{5} \mathrm{~K}$.

At this temperature, the cooling time is short so the shock should be approximately isothermal; the density constrast between the postshock $\left(n_{1}=200 \mathrm{~cm}^{-3}\right)$ and preshock gas $\left(n_{0}=\right.$ $13 \mathrm{~cm}^{-3}$ ) in an isothermal shock is related to the Mach number by $M 2=n_{1} / n_{0}=15$ whereas the relation for an adiabatic shock is simply $n_{1} / n_{0}=4$ (Dyson \& Williams 1997). If the shock were non-magnetized and fully isothermal then $M 2$ would be at least 100 for a $100 \mathrm{~km} \mathrm{~s}^{-1}$ shock. The value of $M 2 \sim 15$ could imply that the shock is not quite isothermal which would be somewhat surprising given that this is a large evolved remnant. If a strong ordered magnetic field was present in the preshock gas then the swept up magnetic field would limit the total compression in the postshock gas (Raymond et al. 1988) in those directions where the field lines were originally parallel to the shock front.

\section{2. $G 59.8+1.2$}

The newly discovered filamentary and diffuse structures towards this remnant show up as incomplete circular structures in the radio, optical and infrared emission. X-ray emission has not been detected so far. They provide a first evidence on the nature of the emission in the area of the remmant suggesting that this emission originates from shock-heated gas resulting from the interaction of the primary blast wave with interstellar "clouds". A new filamentary structure, unknown up to now, has been detected in the low ionization image of $\mathrm{H} \alpha+[\mathrm{N} \mathrm{II}]$ to the south-east of $\mathrm{G} 59.8+1.2$. However, the $[\mathrm{S} \mathrm{II}] / \mathrm{H} \alpha$ ratio suggest that it is an H II structure without any relation to this remnant (Table 2). It is also situated well outside the radio contours of the remnant. The spectrum of G $59.8+1.2$ indicates that the observed shock structures are complete since the $[\mathrm{O}$ III] $] / \mathrm{H} \beta<6$ (Raymond et al. 1988). The absence of soft X-ray emission may indicate a low shock temperature and/or a low density of the local interstellar medium. The failure to detect X-ray emission and the high interstellar extinction prevents the determination of the local ISM density and explosion energy. The [O III] flux production depends mainly upon the shock velocity and the ionization state of the preshocked gas. The absence of [O III] emission cannot help to determine whether slow shocks travel into ionized gas or whether faster shocks travel into neutral gas (Cox \& Raymond 1985) but we can exclude moderate or fast shocks overtaking ionized gas.

Since there are no reliable $\mathrm{H} \beta$ measurements for this remnant, we adopt the statistical relation of Predehl \& Schmitt (1995), using an $E_{B-V}$ calculated from the SFD code. Hence, we obtain an $N_{\mathrm{H}}$ between 1.2 and $2.8 \times 10^{22} \mathrm{~cm}^{-2}$. Furthermore, according to the FTOOLS command "nh" (Dickey \& Lockman 1990), a total galactic $N_{\mathrm{H}}$ value of $1.3 \times 10^{22} \mathrm{~cm}^{-2}$ is measured. However, it is worth mentioning that using the upper limit of $\mathrm{c}(\mathrm{H} \beta)$, calculated from our spectrum, we calculate an $N_{\mathrm{H}}>2.5 \times 10^{22} \mathrm{~cm}^{-2}$ which is in agreement with the above calculations.

Infrared emission (HiRes $60 \mu \mathrm{m}$ ) is found in the area of G 59.8+1.2. In Fig. 5, the optical emission is shown with contours of the IRAS emission where it can be seen that dust is clearly associated with the remnant (Fig. 4). A correlation between the non-thermal radio emission and the infrared is expected (Saken et al. 1992). It is also known that the major, if not the primary, contribution to the infrared emission is from shock-heated interstellar dust swept-up by the supernova shell. Infrared emission is more sensitive to highly evolved remnants, because it can detect shock-heated and radiatively-heated dust in the shells of older remnants. The latter, usually have weak radio, optical, UV and X-ray emission due to low shock velocities but still relatively bright infrared emission (Shull et al. 1989). If this case applies to G $59.8+1.2$, then it could be suggested that it is an evolved remnant.

\section{3. $G 54.4-0.4$}

Like G 59.8+1.2, optical emission is detected for the first time for this remnant. The optical emission cannot define its shape whilst an almost circular structure can be adopted from its nonthermal radio emission. Both optical and radio emission do not uniquely identify the nature of the detected emission, however, the optical observations suggest the existence of shockheated structures. The $[\mathrm{S} \mathrm{II}] / \mathrm{H} \alpha$ ratio of $\sim 0.6$ could be explained by a lower ionization state of the preshocked gas and/or a stronger magnetic field (Raymond 1984; Cox \& Raymond 1985). Almost all ratios of [S II] $\lambda \lambda 6716 / 6731$ are close to the upper end of the allowable range of values suggesting very low electron densities $\left(<50 \mathrm{~cm}^{-3}\right)$. This fact along with the absence of the [O III] emission, the shock modelling of Hartigan et al. (1987) and Raymond et al. (1988) point to shock velocities of $\leq 90 \mathrm{~km} \mathrm{~s}^{-1}$ (probably around 70-80 $\mathrm{km} \mathrm{s}^{-1}$ ) and low preshock clound densities, of the order of a few atoms per $\mathrm{cm}^{3}$.

The interstellar extinction is not accurately determined due to the low significance of the $\mathrm{H} \beta$ flux. However, the lower limits on $c(\mathrm{H} \beta)$, which are derived from our spectra, suggest an area of high interstellar extinction. A value for $N_{\mathrm{H}}$ between 2.9 and $4.0 \times 10^{22} \mathrm{~cm}^{-2}$ is calculated from our data, while the SFD code and the FTOOLS command "nh", result in values of $1.3-3.3 \times 10^{22} \mathrm{~cm}^{-2}$ and $1.5 \times 10^{22} \mathrm{~cm}^{-2}$, respectively. Junkes (1996) using the soft X-ray emission towards G 54.4-0.3, calculated a hydrogen column density of $10^{22} \mathrm{~cm}^{-2}$. Therefore, a value between 1.0 and $4.0 \times 10^{22} \mathrm{~cm}^{-2}$ could be suggested for this remnant. The X-ray emission from G 54.4-0.3 and the surrounding region shows a nice anti-correlation with the cold molecular gas (CO; Junkes 1996). Diffuse X-ray emission is also detected by ASCA in the area of the remnant (Fig. 7) which correlates well with the optical emission found in the west. 
IRAS and high resolution $\mathrm{CO}$ observations of the surrounding region (Junkes et al. 1992b) show an OB-association, a complex of H II regions and a CO-shell, all at the same distance and an association was made with G 54.4-0.3 suggesting that it is part of an extended complex of young population I objects, where its progenitor star possibly was born. According to Chu (1997), when a supernova event occurs in a hot, lowdensity medium as is probably the case, $\mathrm{X}$-ray diffuse emission is expected to be present. This is the case for supernovae with massive stars progenitors which are usually formed in groups, such as OB associations. Therefore, the current X-ray, optical, radio and $\mathrm{CO}$ information give rise to the possibility that this remnant lies within an $\mathrm{OB}$ association.

\section{Conclusions}

Three supernova remnants were observed in major optical emission lines. Emission was discovered for the first time in $\mathrm{G} 59.8+1.2$ and $\mathrm{G} 54.4-0.3$, while previously unknown filamentary and diffuse structures have been discovered in G 126.2+1.6. In all cases, the optical emission is well correlated with the non-thermal radio emission while infrared emission which might be related to the remnants was found only in G 59.8+1.2. Diffuse X-ray emission (ASCA) is also detected in the area of G 54.4-0.3 which probably associates with the optical line emission, while in the case of G 126.2+1.6, X-ray (ROSAT) emission was also found in the area but in order to reach definite conclusions about its association to the remnant, new X-ray observations are needed. No X-ray emission was detected in the area of G 59.8+1.2. The images and the long-slit spectra indicate that the emission arises from shock heated gas.

Acknowledgements. The authors would like to thank the referee (J. Raymond) for his comments and suggestions. We would also like to thank Eric Gotthelf who kindly provided us the ASCA image of G 54.4-0.3 in FITS format. Skinakas Observatory is a collaborative project of the University of Crete, the Foundation for Research and Technology-Hellas and the Max-Planck-Institut für Extraterrestrische Physik. This research has made use of data obtained through the High Energy Astrophysics Science Archive Research Center Online Service, provided by the NASA/Goddard Space Flight Center.

\section{References}

Aumann, H. H., Fowler, J. W., \& Melnyk, M. 1990, AJ, 99, 1674 Blair, W. P., Kirshner, R. P., Gull, T. R., Sawyer, D. L., \& Parker, R. A. R. 1980, ApJ, 242, 592

Boumis, P., Mavromatakis, F., \& Paleologou, E. V. 2002, A\&A, 385, 1042

Chu, Y.-H. 1997, AJ, 113, 1815

Claas, J. J., Kaastra, J. S., Smith, A., Peacock, A., \& de Korte, P. A. J. 1989, ApJ, 337, 399
Cox, D. P., \& Raymond, J. C. 1985, ApJ, 298, 651

Dickey, J. M., \& Lockman, F. J. 1990, ARA\&A, 28, 215

Dopita, M. A. 1979, ApJS, 40, 455

Dyson, J. E., \& Williams, D. A. 1997, The physics of the interstellar medium, 2nd ed. (Bristol: IOP Publishing Ltd)

Fesen, R. A., Gull, T. R., \& Ketelsen, D. A. 1983, ApJS, 51, 337

Fesen, R. A., Winkler, P. F., Rathore, Y., et al. 1997, AJ, 113, 767

Fürst, E., Reich, W., \& Steube, R. 1984, A\&A, 133, 11

Gotthelf, E. V. 2001, An Atlas of ASCA Supernova Remnants, Columbia Astrophysics Laboratory, Columbia University, New York, USA

Green, D. A. 2004, A Catalog of Galactic Supernova Remnants (2004 January version), Mullard Radio Astronomy Observatory Cambridge

Hamuy, M., Walker, A. R., Suntzeff, N. B., et al. 1992, PASP, 104, 533

Hartigan, P., Raymond, J., \& Hartmann, L. 1987, ApJ, 316, 323

Hester, J. J. 1987, ApJ, 314, 187

Holden, D. J., \& Caswell, J. L. 1969, MNRAS, 143, 407

Joncas, G., Roger, R. S., \& Dewdney, P. E. 1989, A\&A, 219, 303

Junkes, N. 1996, in Unsolved problems of the Milky Way, ed. L. Blitz, \& P. Teuben (Cambridge Univ. Press), IAU Symp., 169, 627

Junkes, N., Fürst, E., \& Reich W. 1992a, A\&AS, 96, 1

Junkes, N., Fürst, E., \& Reich W. 1992b, A\&A, 261, 289

Lasker, B. M., Russel, J. N., \& Jenkner, H. 1999, in the HST Guide Star Catalog, version 1.1-ACT, The Association of Universities for Research in Astronomy, Inc.

Mavromatakis, F., Boumis, P., \& Paleologou, E. V. 2002a, A\&A, 383, 1011

Mavromatakis, F., Boumis, P., Papamastorakis, J., \& Ventura, J. 2002b, A\&A, 388, 355

Osterbrock, D. E. 1989, Astrophysics of gaseous nebulae (W. H. Freeman \& Company)

Predehl, P., \& Schmitt, J. H. M. M. 1995, A\&A, 293, 889

Reich, W., Kallas, E., \& Steube, R. 1979, A\&A, 78, L13

Reich, W., Fürst E., Reich P., \& Junkes, N. 1988, in Supernova Remnants and the Interstellar Medium, ed. R. S. Roger, \& T. L. Landecker (Cambridge Univ. Press), IAU Coll., 101, 293

Reich, W., Fürst, E., Reich, P., \& Reif, K. 1990, A\&AS, 85, 633

Reich, W., Zhang, X., \& Fürst, E. 2003, A\&A, 408, 961

Raymond, J. C. 1979, ApJS, 39, 1

Raymond, J. C. 1984, ARA\&A, 22, 75

Raymond, J. C., Hester, J. J., Cox, D., et al. 1988, ApJ, 324, 869

Rosado, M. 1982, RMAA, 5, 127

Saken, J. M., Fesen, R. A., \& Shull, J. M. 1992, ApJS, 81, 715

Schlegel, D. J., Finkbeiner, D. P., \& Davis, M. 1998, ApJ, 500, 525 (SFD)

Shaw, R. A., \& Dufour, R. J. 1995, PASP, 107, 896

Shull, J. M., Fesen, R. A., \& Saken, J. M. 1989, ApJ, 346, 860

Smith, R. C., Kirshner, R. P., Blair, W. P., Long, K. S., \& Winkler, P. F. 1993, ApJ, 407, 564

Xilouris, K. M., Papamastorakis, J., Paleologou, E. V. Andreadakis, Y., \& Haerendel, G. 1993, A\&A, 270, 393 